\title{
Image Case: Solitary Rectal Ulcer Syndrome in a 10 Years Old Boy
}

\author{
Tarik Zaher \\ Tropical Medicine Department, Faculty of Medicine, Zagazig University, Egypt
}

Solitary rectal ulcer syndrome (SRUS) is an uncommon but troublesome and easily misdiagnosed condition of childhood [1]. It is often related to prolonged excessive straining or abnormal defecation and clinically presents as rectal bleeding, copious mucus discharge, feeling of incomplete defecation, and rarely rectal prolapse. SRUS is diagnosed based on clinical symptoms and endoscopic and histological findings [2].

In this case a 10 years old Egyptian boy presented by bleeding per rectum and was examined by colonoscopy which revealed solitary rectal ulcer , hyperemic rectal mucosa, superficial ulceration and hypertrophied rectal folds (SRUS is a misnomer).The boy was treated by laxative and oral mesalamine .
The current treatments are suboptimal, and despite correct diagnosis, outcomes can be unsatisfactory. Some treatment protocols for SRUS include conservative management such as family reassurance, regulation of toilet habits, avoidance of straining, encouragement of a high-fiber diet, topical treatments with salicylate, sulfasalazine, steroids, sucralfate, and surgery[2].

\section{References:}

1.Blackburn C, McDermott M, Bourke B. Clinical presentation of and outcome for solitary rectal ulcer syndrome in children. $J$ Pediatr Gastroenterol Nutr. $2012 ; 54(2): 263-5$.

2.Dehghani SM, Malekpour A, Haghighat M. Solitary rectal ulcer syndrome in children: a literature review. World J Gastroenterol. 2012 ; 7;18(45):6541-5.

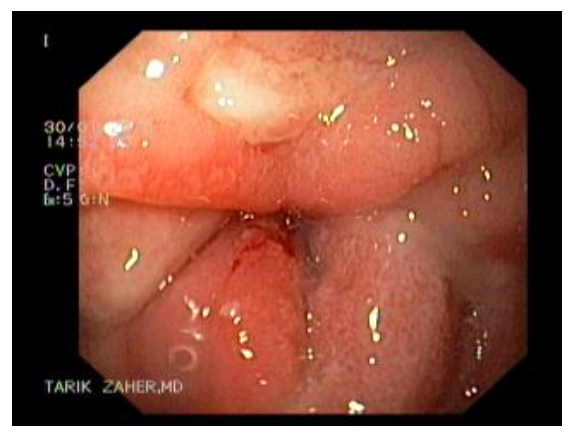

Figure 1: Solitary rectal ulcer

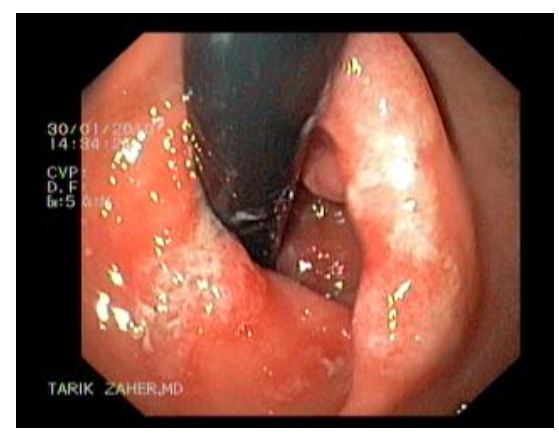

Figure 2: Hypertrophied rectal folds with hyperaemia and superficial ulcers 


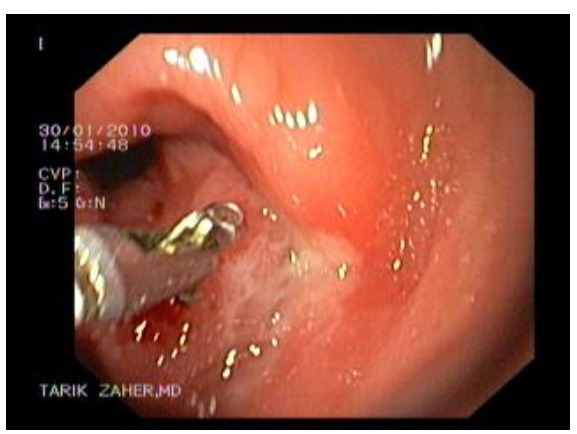

Figure 1:Biopsy of the rectal lesions 